\title{
JOP

\section{Moda e identidade cultural: contribuições do designer para a cultura local}

\author{
Cristiane Rodrigues Neves ${ }^{1}$; \\ Edson José Carpintero Rezende ${ }^{2}$
}

\begin{abstract}
Resumo
A história da moda não é algo frívolo como possa parecer a princípio, uma vez que se pode levantar questões sobre a história da civilização humana no que diz respeito ao clima, aos materiais disponíveis, hierarquia social, assim como identidade cultural. Nesse sentido, é uma importante ferramenta de comunicação, contudo por muito tempo a moda foi exclusiva de realezas e impérios, mais tarde com o poder econômico e político da burguesia a moda passou a ser baseada nos gostos e valores estéticos dessa classe que contava com um estilo urbano, em contraste com os trajes populares associados à vida simples dos trabalhadores do campo. A importância da roupa transcende o tempo, culturas e espaços geográficos, indica quem somos como indivíduos e membros de uma sociedade; alguns povos têm forte relação com suas vestimentas tradicionais. Alguns adornos são considerados símbolos de identidade local, tais adornos possuem valor histórico, muitos dos quais são produzidos pelos próprios membros dessas sociedades; muitas dessas técnicas artesanais ancestrais são passadas de geração para geração, o que proporciona senso de pertencimento e respeito. Porém a indústria do vestuário em massa tem posto em risco este patrimônio em muitas comunidades no mundo, várias casas dedicadas ao artesanato como forma de renda e preservação do patrimônio imaterial estão fechando as portas. Em um momento de distanciamento social devido ao Covid-19, observou-se que o patrimônio imaterial exerce importante papel social, econômico e psicológico na vida das pessoas, visto que muitas comunidades em todo o mundo continuam a produzir seu patrimônio imaterial de forma criativa.

Assim, o objetivo deste estudo é destacar a importância do trabalho do designer para a valorização do artesanato tradicional local como forma de promover emprego e renda e inclusão social desses artesãos. $O$ estudo foi realizado por meio de uma pesquisa bibliográfica que considerou artigos, livros e sites da internet que versam sobre design, moda, sustentabilidade, patrimônio imaterial e identidade cultural. A pesquisa observou exemplos de projetos de designers para o reconhecimento da cultura e tradições locais, a exemplo do trabalho de reconhecimento da importância do tecido kente, para os povos africanos e afrodescendentes promovido por uma marca de moda de Gana em que mescla design contemporâneo com o tecido kente, assim como, o projeto Co-design, cooperação entre designers e artesãos de Portugal para o reconhecimento da importância do casaco "capucha" dos pastores do Norte do país para a história e cultura portuguesa, e ainda, o projeto de valorização da cultura e identidade das mulheres cholas da Bolívia, por meio do trabalho de uma designer local em que elevou o significado desses trajes como valor histórico e cultural do seu país. Observou-se que o trabalho do designer para o reconhecimento da identidade cultural, bem como para a valorização do ofício de artesãos locais é algo possível de ser realizado. Os exemplos em questão apontaram que o trabalho do designer representa uma ampla ferramenta que pode contribuir tanto para a geração de emprego e inclusão social, como para a valorização e preservação dos saberes e valores culturais.
\end{abstract}

Palavras-chave: Moda; Design; Identidade cultural; Patrimônio imaterial.

\footnotetext{
${ }^{1}$ http://lattes.cnpq.br/1902800066658445

2 http://lattes.cnpq.br/5378816399196803
} 


\section{JOP \\ DESIGN \\ de Pós-Graduação em Design - UFMA}

\section{Introdução}

A história da moda não é algo frívolo como possa parecer a princípio, uma vez que se pode levantar questões sobre a história da civilização humana no que diz respeito ao clima, aos materiais disponíveis, hierarquia social, bem como, identidade cultural. Por meio das vestimentas obtém-se pistas sobre o modo de vida dos antepassados: como viveram e trabalharam, suas crenças e valores (HRISTOVA, 2014).

Por muito tempo a moda foi exclusiva das realezas e impérios. Nero, imperador de Roma, baixou um decreto em que tornava a cor púrpura de uso exclusivo do imperador - visto que essa cor era obtida de um corante extraído de um molusco de grande valor econômico, símbolo de riqueza e distinção - a desobediência seria punida com pena de morte (ARAÚJO, 2007; GILL, 2018). Semelhante ao imperador, a rainha Elizabeth, da dinastia Tudor da Inglaterra, em 1562 proibiu o uso de tecidos tintos com a cor púrpura ou bordados com fios de ouro, direito reservado apenas a reis e rainhas (GREENBLATT; MORRILL, 2018; SHULMAN, 2007).

No século XIX, com a revolução industrial e, consequente crescimento do poder econômico e político da burguesia, a moda passou a ser baseada nos gostos e valores estéticos dessa classe. Por esse motivo, a historiografia da moda foi baseada nas classes dominantes, que contavam com um estilo urbano, em contraste com os trajes populares associados à vida simples dos trabalhadores do campo. (ALBURQUERQUE; DELGADO, 2015).

O termo moda apareceu pela primeira vez em 1482, em língua francesa, La nouvelle mode, com a finalidade de introduzir novas tendências ao guarda-roupa da elite. Aos poucos a moda foi se desenvolvendo; no início do século XX começa a surgir os primeiros grandes costureiros, até chegar a uma moda mais acessível a partir dos anos 1980 (DITTY, 2015; HRISTOVA, 2014).

A importância da roupa transcende o tempo, culturas e espaços geográficos; as vestimentas indicam quem somos como indivíduos e membros de uma sociedade. Alguns antropólogos se referem à roupa como uma pele social (SPERANZA, 2019). Por ser considerado um produto socioeconômico e cultural, abrange vários níveis como político, conceitual, representativo assim como de comportamento, estilo e imagem. Nesse sentido, a moda e a indústria do vestuário estão em um momento de questionamento em relação aos seus ciclos de produção, identidade e valores culturais (BUGG, 2010).

Assim, o tema proposto para este estudo é identidade cultural e a contribuição do designer para a cultura local, para tanto foi elaborada a seguinte questão: como o designer pode contribuir para o reconhecimento do artesanato tradicional, enquanto identidade cultural, como forma de preservação e inclusão social? O objetivo geral é analisar contribuições do designer para a preservação e reconhecimento da identidade cultural e seus resultados para a sociedade. O objetivo específico é destacar a importância do trabalho do designer para a cultura local.

Desse modo, optou-se por fazer um estudo sobre o tecido kente de Gana, o casaco "capucha" dos pastores do norte de Portugal bem como sobre os trajes das mulheres cholas da Bolívia, por esses trajes serem historicamente reconhecidos como identidade cultural local. Foram observados exemplos de contribuições de designers locais para a preservação e reconhecimento do valor cultural dessas vestimentas. O método de estudo consistiu em uma busca na literatura que considerou artigos, livros e sites da internet sobre design, moda, identidade cultural, sustentabilidade e patrimônio imaterial.

\section{Moda e identidade}

A maneira como as pessoas se vestem pode ser entendida como uma identidade social formada por valores que todos possuem (HRISTOVA, 2014). Nesse sentido, nosso estilo envia mensagens para o mundo exterior, cada acessório ou peça de roupa é nosso personagem lido pelos outros (DITTY, 2015; 


\section{JOP 21 DESIGN

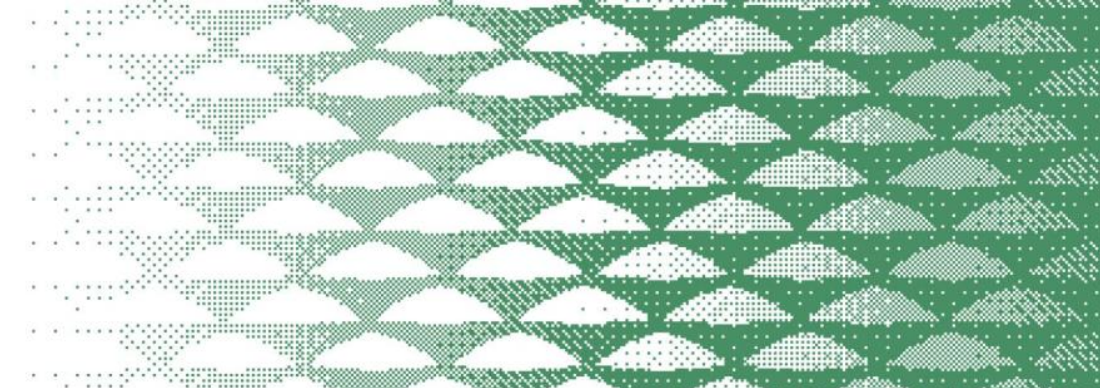

HRISTOVA, 2014; VENKATASAMY, 2018). As escolhas que fazemos estão relacionadas aos signos, à cultura e ao universo de cada um; os significados dados a certos elementos são resultados da relação da percepção com o sistema formado pela cultura, experiências ambientais e memória (MARTINS; ORTUÑO; SANCHES, 2015).

Estudos do campo da sociologia apontaram que a moda possui representação própria no imaginário social, a partir de identificações e concepções referentes às roupas e aos estilos. Esse imaginário tem evoluído, permitindo o desenvolvimento das relações sociais, uma vez que as pessoas se relacionam com o vestir não somente a partir de suas próprias experiências, mas também, a partir das imagens que antecipam uma experiência e que geram uma identidade e uma mensagem que emana do emissor (GOFFMAN, 1959 apud URÍA, 2016).

Os valores simbólicos dados a determinadas roupas e estilos variam em diferentes momentos, visto que o sistema da moda tem suas particularidades em países distintos, uma vez que são influenciados pelas artes locais e outras formas de patrimônio do país bem como pela política e características dos grupos étnicos dos quais são compostos (CRANE; BORONE, 2006).

Figura 1: Líderes locais no festival kente $e^{3}$

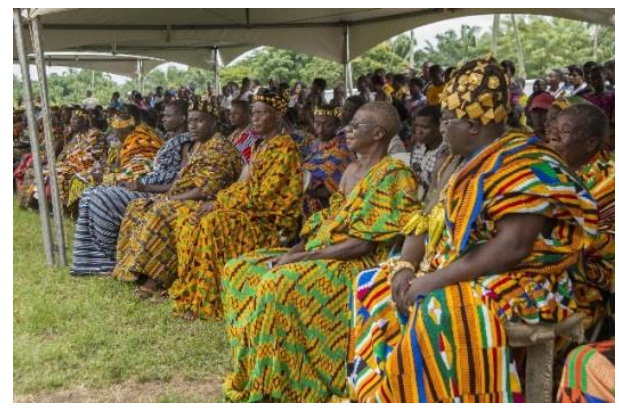

Fonte: https://www.moma.org. Acessado em 14 de jan.2019.

Contudo a permanência de alguns trajes passados de geração para geração demonstra uma receptividade coletiva, em contraste com a tendência atual de extinção dessas vestimentas como resultado da sociedade de consumo (ALBUQUERQUE; DELGADO, 2015). Alguns são reconhecidos como identidade de certos povos, como o tecido kenté ou kente dos povos Asante e Ewé de Gana (FIGURA 1), os trajes das mulheres cholas da Bolívia (FIGURA 2) assim como o casaco "capucha" dos camponeses das regiões montanhosas do norte de Portugal (FIGURA 3).

Figura 2: Cholas bolivianas

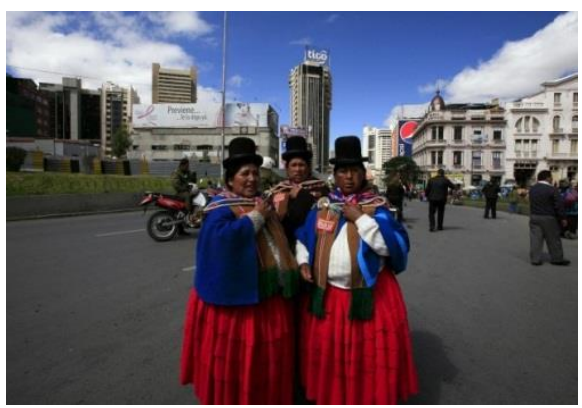

Fonte: http://lifestyle.publico.pt. Acessado em: 14 de jan.2019.

${ }^{3}$ O Festival Kente acontece em julho/agosto a mais de 300 anos para celebrar a origem e importância do tecido kente. $\mathrm{O}$ evento conta com participação de vários líderes e governos locais, com seus corpos adornados com o tecido em diferentes cores e padrões, o que torna o evento um dos mais significativos para a cultura africana. 
Figura 3: Mulheres camponesas com casaco tradicional capucha

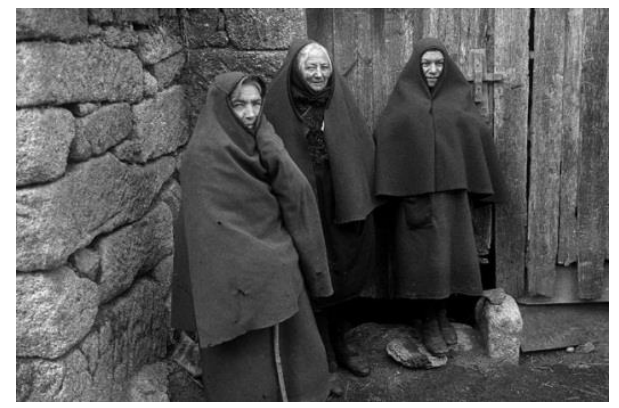

Fonte: https://mood.sapo.pt. Acessado em:14 de jan.2019.

\section{Designer e cultura local}

Segundo a UNESCO (Organização das Nações Unidas para Educação, Ciência e Cultura), o patrimônio imaterial pode ser compreendido como as práticas, representações, habilidades e conhecimentos associados a determinados povos, essas práticas são passadas de geração para geração. Tais práticas e saberes proporcionam senso de identidade e respeito para com a diversidade humana e corrobora para o desenvolvimento sustentável. Dentre os elementos considerados como patrimônio cultural imaterial está incluído o artesanato tradicional (UNESCO, 2003).

Desde os anos 1980 quando muitas empresas do setor de moda aderiram ao modelo fast fashion4, muitos artesãos têm tido dificuldades de sobrevivência. Várias casas dedicadas ao artesanato como forma de renda e preservação do patrimônio imaterial estão fechando suas portas. As técnicas ancestrais, passadas de geração para geração correm o risco de desaparecerem em várias sociedades ao redor do mundo (DITTY, 2015).

Neste momento além da crise sanitária global devido ao Covid-19, o mundo enfrenta uma grande crise econômica como resultado dessa pandemia, o que levou muitas indústrias e pontos comerciais a fecharem as portas em vários países. Contudo pode ser um momento oportuno para colaborações entre designers e artesãos, ao criar projetos que beneficiam as economias locais, alcançam também a proteção dos produtores (MURRAY-NAG. 2020).

Em meio às restrições e ao distanciamento social observou-se que o patrimônio imaterial exerce importante papel social, econômico e psicológico na vida das pessoas, com capacidade de se adaptar e evoluir, visto que comunidades em todo o mundo continuam a praticar seu patrimônio imaterial de maneira criativa (BAROUN, 2020).

Segundo Albuquerque e Delgado (2015), conhecer o artesanato não é apenas trabalhar o passado, os costumes e tradições, mas, sobretudo, conhecer nossos valores ancestrais e suas técnicas de modo a criar um ambiente de criatividade, tradição, inovação e design que relacione aspectos técnicos e culturais na construção de um patrimônio nacional. $\mathrm{O}$ avivamento de tradições há muito esquecidas colabora para o estudo e o aprendizado de técnicas e métodos esquecidos no tempo assim como desvela às próximas gerações o modo de vida de seus ancestrais (TEIXEIRA, 2018).

De acordo com Todorovic et al. (2014), atualmente os trajes típicos são vistos como símbolos de reconhecimento de nações, cultura e linguagem de vários povos. A recuperação de velhas formas tradicionais e abordagens para o vestuário tem o propósito de transmitir para as futuras gerações a preservação da tradição e enriquecimento da herança cultural.

${ }^{4} \mathrm{O}$ termo fast fashion foi cunhado pela primeira vez pelo The New York Times em 1989 para descrever o modelo de negócios da Zara (cadeia de lojas de moda espanhola) no qual as lojas eram abastecidas com novos modelos a cada duas semanas em vez de 4 coleções, como era conhecido anteriormente (SCHIRO, 1989). 
Contribuições de designers para a valorização do artesanato tradicional, não é algo tão recente. $\mathrm{O}$ interesse atual pela identidade cultural e pelo artesanato tradicional reproduz um movimento denominado Arts and Crafts ocorrido na Europa entre o século XIX e XX. O princípio norteador desse movimento era apoiar a cultura e a produção artesanal local. Muitos artistas e designers adeptos do movimento faziam parcerias com trabalhadores rurais na criação de seus produtos. Lewis Foreman Day (1845-1910), designer industrial e decorador britânico, foi uma figura de destaque deste movimento na Inglaterra (Victoria and Albert Museum, 2019).

O designer cria produtos apropriados e os conecta em um processo denominado fenômeno da moda, contudo esse fenômeno está tornando-se cada vez mais mutável, em um curso muito rápido nesta era do consumo em massa. Assim, os trajes típicos evocam uma memória pela identidade, em um contexto de adaptação às diferentes necessidades sociais (ALBUQUERQUE, DELGADO, 2015).

Nesse sentido, observa-se o tecido Kente dos povos Asante e Ewé de Gana, que historicamente foi símbolo de riqueza e poder, e com o tempo, passou a fazer parte de várias sociedades africanas. O termo kente, alguns acreditam, ser derivado da palavra de origem fanti: kenten que significa cesto, por sua trama lembrar o entrelaçado dos cestos, outras fontes creditam o termo ao dialeto do povo Ewé: ke (abrir) te (pressionar), kenate é o processo de tecer a fibra, em que abre os fios, passa a trama e pressionam repetidas vezes; com o tempo o termo foi alterado para kente (LLOYD, 2017; MICOTS, 2018; PEZZOLO, 2013).

Em 1957, com a independência de Gana do domínio inglês, seu primeiro presidente Kwame Nkrumah5 adotou o Kente como símbolo do país para promover unidade, orgulho e identidade nacional. Desde então, o Kente tornou-se um símbolo da identidade africana, inclusive para os afroamericanos e africanos que vivem em diáspora (HALLS; MARTINO, 2018; MICOTS, 2018; THE NEW TIMES, 2017).

Figura 4: criação da marca Christie Brown

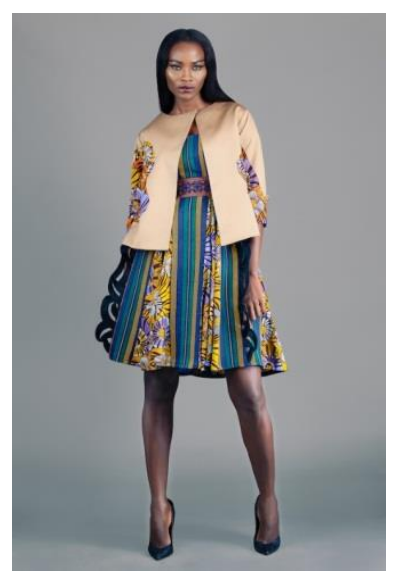

Fonte: https://www.angiebroks.com/fashion. Acessado em: 14 de jan.2019.

Muitos designers de moda do continente africano têm percebido a necessidade de trazer elementos da cultura local para suas produções, como forma de preservar a herança imaterial e valorizar sua cultura (LANGEVANG, 2016). A primeira designer de moda de Gana conhecida internacionalmente por mesclar elementos contemporâneos com tecidos tradicionais foi Juliana Norteye, da marca Chez Julie, nos anos 1950 (RICHARDS, 2015; RICHARDS, 2016).

${ }^{5}$ Kwame Nkrumah (1909-1972) foi um político e líder popular ganense, que lutou pela independência de Gana. Em 1954 torna-se primeiro ministro. Três anos mais tarde, conquista a independência do seu país, em 1960 tornase presidente. Gana esteve sob o domínio inglês de 1807 até 1957 (BRITISH BROADCASTING COMPANY, 2018). 


\section{JOP \\ DESIGN \\ de Pós-Graduação em Design - UFMA}

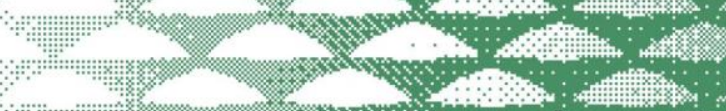

Atualmente a marca Christie Brown (FIGURA 4) ficou conhecida internacionalmente por exaltar a identidade africana ao trazer símbolos locais como estampas vibrantes, artesanatos tradicionais e tecidos africanos ancestrais, mesclando-os com outros elementos de uma forma elegante e contemporânea. Além de representar a riqueza da cultura e do artesanato africano, a marca observa padrões éticos e sustentáveis, visto que a manufatura, os tecidos e outras matérias-primas são providos de fornecedores locais ou de outros países do continente africano, o que gera empregos para artesãos, costureiros e designers locais (HIRSCH, 2019; JENNINGS, 2011).

Em Portugal, há um projeto denominado co-design, iniciado no final dos anos 1990. O principal objetivo é a inovação e sustentabilidade econômica local assim como o reconhecimento da importância histórica e cultural do casaco "capucha" dos pastores e camponeses do norte de Portugal. Designers e membros de uma cooperativa dedicada à arte tradicional da tecelagem de lã e linho, na cidade de Campo Benfeito, norte do país, têm trabalhado juntos na confecção de várias peças, porém o item mais significativo é o casaco tradicional capucha (FIGURA 5); confeccionado em design contemporâneo, contudo, observando as técnicas e materiais tradicionais. O projeto trouxe viabilidade econômica e inclusão social ao valorizar os conhecimentos ancestrais dos artesãos (ALBUQUERQUE, DELGADO, 2015; SILVA, 2010).

Esse casaco é símbolo dos camponeses das regiões montanhosas do norte de Portugal, como Trás-os-Montes e Serra da Estrela, feito de burel (lã prensada), de estilo sóbrio, porém confeccionado com técnica exemplar para suportar o clima frio e seco das regiões montanhosas do norte do país (TEIXEIRA, 2018).

Figura 5: casaco capucha em design contemporâneo
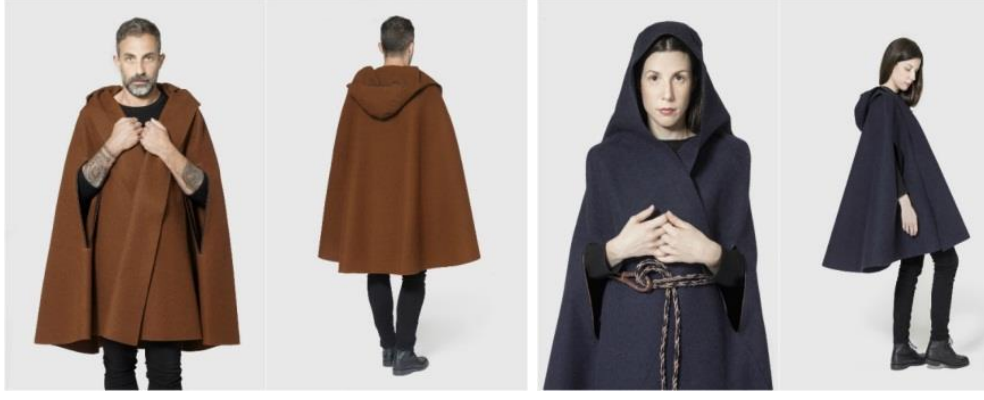

Fonte: https://mood.sapo.pt. Acessado em: 14 de jan.2019.

Em La Paz, Bolívia, as Cholas (FIGURA 6), mulheres indígenas, são há muito tempo estigmatizadas pela sociedade local, por usarem seus trajes típicos: chapéus-coco, xales de macramê, blusas feitas sob medida, saias longas e vários adornos elaborados. Embora o estilo chola tenha sido declarado em 2013 herança cultural da capital do país, La Paz, muitas dessas mulheres são, por vezes, impedidas de frequentarem certos locais públicos, que as associam à uma subclasse de mulheres operárias. (FASHION UNITED, 2016; HERALD TRIBUNE, 2019; LITTLE, 2016).

Há um consenso, de que o termo chola é oriundo da Península Ibérica, cholo (rufião) era o nome dado ao ajudante do toureiro, sua esposa, a chula, usava uma saia longa e plissada com blusa decorada e xale bordado. Além de várias outras especulações, o termo cholo foi estendido pejorativamente pelos espanhóis "puros" em relação aos "não puros", particularmente, os indígenas que falavam espanhol e vestiam trajes semelhantes aos espanhóis (GARCÍA, 2011).

Para a mulher chola, a moda tem sido um reflexo social de identidade. Por meio de suas roupas ela mostra que faz parte de um grupo social dentro de um meio essencialmente dominado pela estética convencional, entendida como o estilo ocidental (LITTLE, 2016; MACEDA, 2016). 


\section{JOP' \\ DESIGN \\ de Pós-Graduação em Design - UFMA}

Nos últimos anos, a designer de moda boliviana Eliana Paco Paredes tem mitigado o estigma do estilo chola por meio de suas criações já mostradas na semana de moda de Nova Iorque, ao mesmo tempo em que contribui para a sustentabilidade econômica dos artesãos locais, por suas criações utilizarem, dentre outros materiais, tecidos tradicionais bolivianos como a bayeta de la tierra (tecido de lã do Altiplano, região central da Bolívia) e o aguayo (tecido feito por artesãos e mulheres indígenas) (HERALD TRIBUNE, 2019).

Suas roupas podem ser consideradas um grande negócio, uma vez que esses trajes têm sido depreciados na Bolívia por serem historicamente usados por mulheres pobres e indígenas. $\mathrm{O}$ reconhecimento internacional do design de Paredes poderá aumentar a aceitação das mulheres indígenas e sua cultura na Bolívia (LITTLE, 2016).

Figura 6: Cholas, desfile de Eliana Paco Paredes

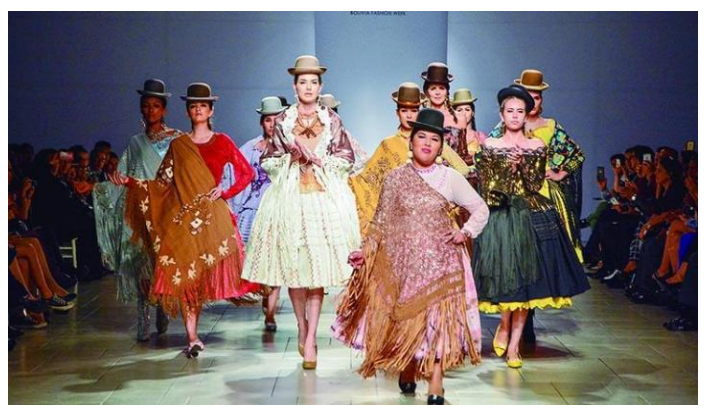

Fonte: https://voguetalks.wordpress. Acessado em: 14 de jan.2019.

\section{Considerações finais}

Observou-se por meio deste estudo que o artesanato reproduz uma riqueza imaterial que representa diferentes povos na terra, o que lhes confere senso de pertencimento e identidade. A importância da preservação desse patrimônio abrange os aspectos social, econômico e humano além de contribuir para a sustentabilidade, uma vez que sua continuidade constitui uma forma de preservação de emprego e renda para muitos artesãos, o que chamou a atenção de artistas e designers para o desenvolvimento de projetos de parceria com artesãos locais.

Os resultados mostraram que é possível valorizar a cultura e o artesanato tradicional, assim como fomentar a economia local, a exemplo de parcerias entre designers e artesãos observado no projeto co-design de Portugal. Percebeu-se o papel do designer como sujeito social, quando por meio de seu trabalho contribui para valorização de grupos étnicos marginalizados, tendo como exemplo as mulheres cholas da Bolívia, que têm conquistado reconhecimento internacional e inclusão social em seu país de origem por meio do design de Paredes. A importância histórica do tecido kente para a cultura africana há muito tempo é reconhecido internacionalmente, porém o trabalho de designers locais tem buscado valorizar a identidade local de uma forma inovadora, em que mescla design contemporâneo com artesanato e tecidos tradicionais.

Neste estudo observou-se que o designer procurou elevar o valor e reconhecimento da cultura local, ao mesmo tempo em que contribuiu com soluções sustentáveis quando da recuperação e preservação de técnicas ancestrais, gerando emprego e renda para as populações locais. Percebe-se que o designer, por meio de seu ofício, representa uma ampla ferramenta que pode contribuir grandemente para o bem-estar social.

O Brasil, por ser um país multicultural, conta com uma rica variedade de artesanatos, muitos dos quais têm como base os têxteis que empregam técnicas de costura passadas de geração para geração. Em tempos de grandes incertezas econômicas devido à pandemia do Covid-19, poderia ser o 
momento oportuno para designers reconhecerem e valorizarem a identidade cultural, assim como contribuírem para a geração de emprego e inclusão social de artesãos e artistas locais.

\section{Referências}

AYMARA Designer Showcases Lastest Collection at Bolívia's Presidential Palace. Herald Tribune- Latin American. Disponível em: http://www.laht.com/article.asp?ArticleId=2416569\&CategoryId=14919. Acesso em 16 de jan. 2019.

ARAÚJO, E. M. de. Corantes naturais para têxteis-da antiguidade aos tempos modernos. Conservar Património, Lisboa, $\mathrm{n}$. 3 e $4, \quad$ p. $37 \quad-\quad 49, \quad 2006 \quad / \quad 2007$. Disponível em:http://moodlearquivo.ciencias.ulisboa.pt/1314/pluginfile.php/83484/mod_resource/content/1/4_corantes.pdf. Acesso em 02 out. 2018.

BOLIVIAN Designer Exports High-end Indigenous Fashion. FASHION UNITED. 14 nov. 2016. Disponível em: https://fashionunited.uk/news/fashion/bolivian-designer-exports-high-end-indigenous-fashion/. Acesso em: 23 dez. 2018.

BAROUN, A. How Covid-19 is affecting our intangible heritage. Patrimonie d'Orient Observatory. 20 de jul. 2020. Disponível em: https://patrimoinedorient.org/index.php/en/2020/07/29/how-covid-19-is-affecting-our-intangible-heritage/. Acesso em: 29 de maio 2021.

BUGGS, J. The shifting focus: culture, fashion and identity. In: 24th INTERNATIONAL COSTUME CONGRESS: FASHION, CULTURE AND IDENTITY, 24 a 25 de agosto de 2010, Seoul. Anais...National Museum of Korea in Collaboration with Yonsei University, Seoul, Korea.2010, p.9. Disponível em: http://ualresearchonline.arts.ac.uk/4945/. Acesso em 09 nov. 2018.

CRANE, D.; BOVONE, L. Approaches to Material Culture: the Sociology of Fashion and Clothing. Science Direct [S.I.] 15p. 2018. Disponível em: https://www.sciencedirect.com/science/article/pii/S0304422X06000428. Acesso em 09 nov.2018.

DITTY, S. It's time for fashion revolution. White Paper, [S.I.] p.1-29, dez, 2015. Disponível em: https://www.fashionrevolution.org/wp-content/uploads/2016/08/FashRev_Whitepaper_Dec2015_screen.pdf. Acesso em: 12 nov. 2018.

DELGADO, M.J.B.F.; ALBUQUERQUE, M.H.F. The contribution of regional costume in fashion. In: 6th INTERNATIONAL CONFERENCE ON APPLIED HUMAN FACTORS AND ERGONOMICS AND THE AFFILIATED CONFERENCES. 8p. 2015. Disponível em: https://www.sciencedirect.com/science/article/pii/S2351978915009671. Acesso em 09 nov. 2018.

GARCÍA, H. R. Mestizage y Conflictos Sociales. El Caso de la Construcción Nacional Boliviana. CUADERNOS INTERCAMBIO. Ano 8 n.9 p.145-182, São Pedro de Montes de Oca, 2011. Disponível em: https://www.redalyc.org/articulo.oa?id=476948771008. Acesso em 09 dez. 2018.

GHANA country profile. BBC-news. 01 de mai. 2018. Disponível em: https://www.bbc.com/news/world-africa-13433790. Acesso em 14 de jan. 2019

Gill, N.S. Profile of the Roman Emperor Nero. ThoughtCo. History and Culture. 03 de mar. 2018. Disponível em: https://www.thoughtco.com/all-about-nero-119988. Acesso em: 19 de dez. 2019.

HALLS, J.; MARTINO, A. Cloth, Copyright, and Cultural Exchange: Textile Designs for Export to Africa at National Archives of the UK. Journal of Design History, v.31, n.3, p.236-254, set, 2018. Disponível em: https://academic.oup.com/jdh/article/31/3/236/4924337. Acesso em 10 dez. 2018.

HIRSCH, A. African Luxury Fashion: Designer Ayensu. BBC World Service. 16 de jan. 2019. Disponível em: https://www.bbc.co.uk/programmes. Acesso em 16 jan. 2019.

HOW Arts and Crafts influenced fashion. V\&A- Victoria and Albert Museum. London, 2019. Disponível em: https://www.vam.ac.uk/articles/how-arts-and-crafts-influenced-fashion. Acesso em: 14 de jan. 2019.

HRISTOVA, T. Clothing - a Choice and Image of Cultural Identity. Postmodernism Problems. v.4, n.1, 8p, [S.I.] 2014. Disponível em: http://ppm.swu.bg/media/39414/hristova_t.cloting-a_chois_and_image\%206.pdf. Acesso em 17 nov. 2018.

JENNINGS, H. Why African Designers are Finally in the Fashion Spotlight CNN Business. nov. 2011. Disponível em: https://edition.cnn.com/2011/11/04/business/helen-jennings-africa-fashion/index.html. Acesso em 20 dez. 2018.

KWAME Nkrumah: Ghana's first president and a revered panafrican. The New Times - Rwanda's Leading Daily. 01 nov. 2017. Disponível em: https://www.newtimes.co.rw Acesso em 15 de jan. 2019.

LANGEVANG, T. Fashioning the Future: Entrepreneuring in Africa's Emerging Fashion Industry. CBDS (Centre for Business and Development Studies). Working Paper Series. n.25 40p.D 2016 . Disponível em: https://link.springer.com/article/10.1057/s41287-016-0066-z. Acesso em: 07 dez. 2018.

LITTLE, B. Indigenous 'chola' Clothing Comes to Fashion Week. National Geografic. Set. 2016. Disponível em: https://news.nationalgeographic.com/2016/09/fashion-week-new-york-chola-clothing-bolivia/. Acesso em 01 out. 2018. 


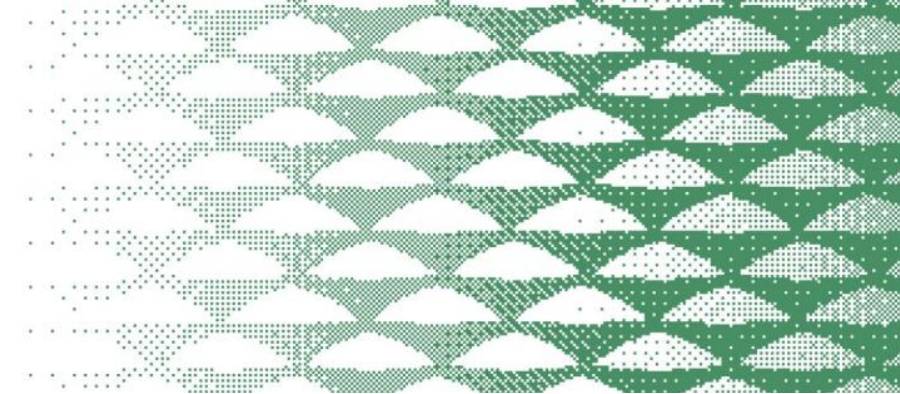

LLOYD, K. Embrace a tradition Royalty. Kente Cloth Origins.

Abr. 2017. Disponível em: https://www.kentecloth.net/kente-cloth-origins/. Acesso em 20 dez.2018.

MACEDA, V. M. S. Cholas fashionistas, cuando la identidad se porta em la pollera, la manta y el sombrero. In: XIII FORO ACADÉMICO DE DISEÑO- Festival internacional de la imagem. 2016, Manizales, Colômbia. Anais... Manizales, Colômbia. 9-13 mai. 2016. 13p. Disponível em: http://festivaldelaimagen.com/wpcontent/uploads/2017/07/Valeria_Salinas.pdf. Acesso em: 10 dez. 2018.

MARTINS, S. R. M.; ORTUÑO, B. H.; SANCHES, M. C. F. Fashion Design: The Project of Intangible. In: 6th INTERNATIONAL CONFERENCE ON APPLIED HUMAN FACTORS AND ERGONOMICS AND THE AFFILIATED CONFERENCES. 8p. 2015.Disponível em: https://www.sciencedirect.com/science/article/pii/S2351978915003789. Acesso em: 09 nov. 2018.

MICOTS, C. Kente Cloth (Asante and Ewe Peoples). Khan Academy. [S.I.]. Disponível em: https://www.khanacademy.org/humanities/art-africa/west-africa/ghana/a/linguist-staff-okyeamepoma-asante-peoples. Acesso em: 01 nov.2018.

MORRIL, J.S.; GREENBLATT, S.J. Elizabeth I, Queen of England. Encyclopaedia Britannica. 02 nov. 2018 Disponível em: https://www.britannica.com/biography/Elizabeth-I. Acesso em: 19 dez. 2018.

MURRAY-NAG, B. Sustainability Stories "We Created Before and We'll Create Again”: Preserving. Craftmanship Through a Pandemic. Eco, 2020. Disponível em: https://eco-age.com/resources/preserving-artisan-craftsmanship-through-covid-19pandemicl. Acesso em: 19 de fev. 2021.

PEZZOLO, D. B. Tecidos: histórias, tramas, tipos e usos. 4. ed. São Paulo: Editora Senac São Paulo,2013.328 p.

RICHARDS, C. Kabas and Couture- Contemporary Ghanaian Fashion. Museum of Art- University of Florida, 16p. Gainsville, 2015. Disponível em: http://www.harn.ufl.edu/linkedfiles/publication-kabasbrochure.pdf. Acesso em: 07 dez.2018.

RICHARDS, C. Models for Africa. Accra's Indenpendence Era- Fashion Culture and the Creation of Chez Julie. AFRICAN ARTS. V.49 N.3, 2016. Disponível em: https://www.mitpressjournals.org/doi/pdf/10.1162/AFAR_a_00296. Acesso em: 20 dez. 2018.

SCHIRO, A. M. Fashion; two new stores that cruise fashion's fast lane. 31 dez. 1989. The New York Times. Disponível em: https://www.nytimes.com/1989/12/31/style/fashion-two-new-stores-that-cruise-fashion-s-fast-lane.html. Acesso em: 29 maio 2021.

SILVA, H. S. M. S. Glocalness: Identidade e Memória no Design Português Contemporâneo. Dissertação de Mestrado Escola Superior de Artes e Design. 121p. [S.I.] 2010. Disponível em: https://comum.rcaap.pt/bitstream/10400.26/6925/1/TESE_FINAL\%20CD.pdf. Acesso em 21 dez. 2018.

SHULMAM, R. Sumptuary Legislation and Fabric Construction of National Identity in Early Modern England. Constructing the Past. v.8, N.1. Bloomington. 2007. Disponível em: https://digitalcommons.iwu.edu/cgi/viewcontent.cgi?article=1006\&context=constructing. Acesso em 10 out. 2018.

SPERANZA, A. Why are clothes so important when It comes to building a sustainable culture? London Community Resource Network. [S.I] [2015?] Disponível em: http://lcrn.org.uk/clothes-important-comes-building-sustainable-culture/. Acesso em 08 de jan. 2019.

TEIXEIRA, M.B. O TRAJE REGIONAL PORTUGUÊS E O FOLCLORE VII. 54p (353-406). [S.I] [2015?] Disponível em: https://www.om.acm.gov.pt/documents/58428/182327/1_PI_Cap7.pdf/803cd114-b7e8-448a-8dea-aeacb67866fe. Acesso em 05 dez.2018

TODOROVIC, T.; TOPORISIC, T.; CUDEN, A. Clothes and Costumes as Form of Nonverbal Communication. Tekstilec, Letn. n. 57, v. 4, p. 321-333, Liubliana, 2014. Disponível em: http://www.tekstilec.si/wp-content/uploads/2014/12/321333.pdf. Acesso em: 08 de jan. 2019.

UNESCO (Organização das Nações Unidas para Educação, Ciência e Cultura) Convention for the Safeguarding of Intangible Cultural Heritage. Paris: UNESCO, 2003. Disponível em: https://unesdoc.unesco.org/ark:/48223/pf0000132540. Acesso em 02 dez. 2018.

URÍA, A. S. C. La Identidad a Través de La Moda. Revista Humanidades. n. 29, p.131-152. Sevilha 2016. Disponível em: http://revistas.uned.es/index.php/rdh/article/view/17220. Acesso em: 02 out. 2018.

VENKATASAMY, N. Fashion trends and their impact on the society. In: CONFERENCE ON TEXTILE, APPARELS AND FASHION, 2015, Coimbatore. Disponível em: https://www.researchgate.net/publication/282571020_Fashion_trends_and_their_impact_on_the_society. Acesso em: 16 nov. 2018. 